\title{
LA NECESIDAD DE PROMOVER EL DEBATE EPISTEMOLOGICO DE LA PEDAGOGÍA EN LAS FACULTADES DE CIENCIAS DE LA EDUCACION, DESDE LA PERSPECTIVA LATINOAMERICANA
}

\section{THE NEED TO PROMOTE THE EPISTEMOLOGICAL DEBATE OF PEDAGOGY IN THE FACULTIES OF EDUCATION SCIENCES, FROM THE LATIN AMERICAN PERSPECTIVE}

Mirella Del Pilar Vera-Rojas ${ }^{1}$ Facultad de Ciencias de la Educación, Humanas y Tecnologías Universidad Nacional de Chimborazo, Riobamba, Ecuador

\section{Luis Fernando Chávez Vera ${ }^{2}$ Carrera de Enfermería}

Instituto Superior Tecnológico "San Gabriel", Riobamba, Ecuador

\section{Luis Antonio Vera Rojas ${ }^{3}$ Facultad de Ciencias}

\section{Escuela Superior Politécnica de Chimborazo, Riobamba, Ecuador}

$1 \quad$ Profesional en Filosofía y Ciencias Socio - Económicas y Doctora en Planeamiento y Diseño Curricular de la Universidad Nacional de Chimborazo, Magister en Docencia Universitaria e Investigación Educativa de la Universidad Nacional de Loja. Docente Investigadora del Instituto de Ciencia, Innovación, Tecnología y Saberes de la UNACH; Vicerrectora Académica del Instituto Tecnológico Superior San Gabriel de la ciudad de Riobamba 2004-2010 y Doctora Ph.D. en Ciencias Pedagógicas por la Universidad de La Habana, Cuba. https://orcid.org/0000-0001-6896-1391

2 Licenciado en Laboratorio Clínico e Histopatológico de la Universidad Nacional de Chimborazo, Profesor y Coordinador del Departamento de Vinculación de la Carrera de Enfermería del Instituto Superior Tecnológico San Gabriel de la ciudad de Riobamba, Ecuador. https://orcid.org/0000-0002-9813-3404

3 Doctor en Matemática y Magister en Matemática Aplicada Mención Modelación Matemática y Simulación Numérica de la Escuela Superior Politécnica de Chimborazo. Director y Profesor de la Carrera de Física y Matemática de la Facultad de Ciencias de la ESPOCH en el que ha dictado las cátedras de Cálculo Matemático y Estadística Descriptiva e Inferencial. https:/l orcid.org/0000-0002-7087-8447 


\section{RESUMEN}

El presente artículo fruto de la revisión de distintas aportaciones científicas de autores latinoamericanos, luego de hacer un breve recorrido histórico de la Educación, la Pedagogía y la escuela como organización social, expone las razones por las que el debate del estatuto epistemológico de la Pedagogía en América Latina debe ser revitalizado por educadores y pedagogos en las Facultades de Ciencias de la Educación, quienes además de formar integralmentealoseducandos, estamos llamados a continuar con el avance y desarrollo de esta ciencia, para empezar a educar a nuestros niños y jóvenes con modelos educativos y pedagógicos propios que respondan a las particularidades, singularidades, intereses y reales necesidades de la región, en función de nuestra particular cultura e historia, y refleje los avances ocurridos en los países que la conforman y brindemos una educación emancipadora que tanto necesitan nuestros pueblos.

PALABRAS CLAVE: Educación, Pedagogía, escuela,epistemologíapedagógica, investigación pedagógica y región latinoamericana.

\section{ABSTRACT}

This article is the result of the review of different scientific contributions of Latin American authors, after making a brief historical tour of Education, Pedagogy and the school as a social organization, exposes the reasons why the debate on the epistemological status of Pedagogy in Latin America must be revitalized by educators and pedagogues in the Faculties of Education Sciences, who in addition to fully educating students, we are called to continue with the advancement and development of this science, to begin educating our children and youth with their own educational and pedagogical models that respond to the particularities, singularities, interests and real needs of the region, depending on our particular culture and history, and reflect the advances that have taken place in the countries that make it up and provide an emancipatory education that our peoples need so much.

KEY WORDS: Education, Pedagogy, school, pedagogical epistemology, pedagogical research and Latin American region.

\section{DESARROLLO}

La educación como práctica social ha estado presente de manera espontánea desde hace 2,85 millones de años a.C con los primeros grupos humanos, reproduciendo y transformando conocimientos y destrezas desde las actividades de subsistencia: caza, pesca y recolección (comunidad primitiva) hasta la sedentarización de los pueblos con la agricultura, pastoreo y alfarería (esclavismo), etapa en la que aparece la educación sistemática presente hasta nuestros días y manifestada con características diferentes también en el feudalismo, capitalismo y socialismo.

En tanto que la Pedagogía es ciencia relativamente joven (fines del siglo XVIII y principios del siglo $X I X)$, estudia la educación en forma intencionada, sistemática y científica para solucionar los problemas que en ella se presentan y a formar integralmente a los sujetos en las diferentes etapas de su vida. De ahí que la historia de la ciencia se ha relacionado con la historia de la humanidad, donde se han ido conformando las ideas y los diferentes preceptos sobre como formar a los individuos dentro de las distintas sociedades y los grupos humanos para que estos sean continuadores del desarrollo de la humanidad.

Las ideas, los conceptos y las acciones sobre estos procesos formativos son en la actualidad los aspectos esenciales del cuerpo epistemológico de la Pedagogía como ciencia, mismo que ha estado determinado por el desarrollo de la sociedad en sus diferentes 
etapas, caracterizado por un conjunto de ideas, sustentadas a partir de la visión empírica o no que se tenga de las características del proceso formativo.

Dicho proceso ha estado mediado por las demandas sociales y por el desarrollo de otras ciencias que desde diferentes perspectivas indagan y fundamentan las relaciones del ser humano con la naturaleza, la sociedad y otros sujetos, lo que ha permitido formar diferentes teorías sobre la educación.

Las teorías son el resultado del pensamiento y la acción de hombres y mujeres que en distintos momentos de la historia de la humanidad han dejado explícitos sus puntos de vistas sobre el hecho educativo, por tanto cualquier aproximación a la Pedagogía como ciencia pasa necesariamente por el estudio del contexto y las obras científicas de quienes han reflexionado sobre ese particular.

En este sentido, las investigaciones pedagógicas constituyen las principales vías de indagación que permiten relacionar el pensamiento, las prácticas educativas y las obras de destacados educadores con el desarrollo epistemológico de la Pedagogía. Al respecto, Buenavilla (2001) ha señalado con verdadero acierto que "se reúnen bajo el concepto de educadores a todas las personalidades que se relacionan de una forma u otra con la educación y los procesos pedagógicos concomitantes. Se incluyen aquí no solo a los que se mueven en la esfera de la escuela y el sistema nacional de educación, sino aquellos que han ejercido una influencia educativa aunque no propiamente escolar" ( $p$. 1-2).

Por lo tanto, el movimiento del pensamiento pedagógico no es lineal ni circular ni pendular, es un proceso dialéctico, que se manifiesta en diferentes fases, donde se reflejan las ideas y los preceptos que van determinando los fundamentos epistemológicos de una ciencia (Gadotti, 1998).

Siguiendo con esta mirada histórica, las primeras nociones de cómo educar se remontan a la sociedad primitiva, donde el niño a decir de Konstantinov (1974) "se educaba e instruía en el proceso de su propia actividad vital, mediante su participación en los asuntos de los mayores, en su contacto diario con ellos" (p. 11), con lo se promovía fundamentalmente la solidaridad y la espontaneidad entre sus miembros.

Más tarde con la división social del trabajo, aparecen las especialidades, la escuela ya no es la aldea o la vida, esta empieza a funcionar en un lugar especializado donde unos aprenden y otros enseñan. La escuela que aparece desde la sociedad esclavista, nació como producto de "la jerarquización y la desigualdad económica generada por aquellos que se apoderaron del excedente producido por la comunidad primitiva. Desde entonces la historia de la educación se constituye en una prolongación de la historia de las desigualdades económicas" (Gadotti, 2003. p. 9).

En la medida que la sociedad se organizó en clases sociales, el pensamiento pedagógico también respondió a las clases que tenían el poder económico y político, iniciándose el proceso de escolarización (señalado en el párrafo anterior) como una forma de organizar la educación, la cual se ha mantenido hasta la actualidad en su esencia; mientras que la Pedagogía como conocimiento autónomo y sistematizado aparece en la segunda mitad del siglo XIX, aunque se reconoce sus antecedentes desde el siglo XVIII, continuando su desarrollo durante todo el siglo $\mathrm{XX}$, particularmente después de la Primera Guerra Mundial (1914 1918).

En 1917 con el triunfo de la revolución rusa cobra gran importancia la educación del pueblo, lo que demandó investigaciones, análisis y reflexiones 
sobre el proceso de educación, contribuyendo con ello al desarrollo de una Pedagogía con enfoque marxista.

Después de finalizada la segunda guerra mundial (mayo de 1945), los países afectados por la confrontación así como los países victoriosos priorizan la educación de las masas como una vía para lograr la reconstrucción, la paz y el desarrollo, lo que provoca un debate alrededor de la Pedagogía como ciencia y su importancia dentro de las ciencias sociales. En este momento es de destacar la propuesta teórica derivada de la Pedagogía MarxistaLeninista sustentada en los países del antiguo campo socialista.

Por otra parte, Estados Unidos al emerger de esta contienda como una potencia mundial, dedica recursos para la formación de sus ciudadanos y su consolidación ideológica en la región y en el mundo. Para ello durante la primera mitad del siglo $X X$ se crean espacios donde la reflexión sobre la Pedagogía se generaliza a través de los principios y postulados de la Escuela Nueva y el Pragmatismo. Estos enfoques sobre la Pedagogía llegan a la región latinoamericana donde no siempre encontraron una mirada analítica y valorativa con respecto a su pertinencia en relación con las necesidades educativas de este contexto.

Las décadas del ochenta y el noventa del siglo XX cambiaron de manera radical el panorama mundial. La caída del campo socialista (1991) y la generalización del neoliberalismo como una propuesta global provocaron una crisis de las ciencias sociales y de los paradigmas marxista, aspecto este que tuvo consecuencias en la Pedagogía, y que es valorado desde diferentes puntos de vista de investigadores de la región.

Según valora Julia Silber (2006) en su artículo, "Reflexiones Epistemológicas sobre la Pedagogía"( https://es.scribd.com/doc/), la Pedagogía ha ido cumpliendo sus misiones en cada época y en cada contexto, pero estas nunca estuvieron alejadas de contradicciones complejas, ya que en algunas circunstancias sometió sus teorizaciones y sus intervenciones al campo filosófico, en otras circunstancias respondieron al poder político, donde se favorecía las "demandas educativas", para a partir de ellas desarrollar las investigaciones, sin desconocer que también fue discriminada por sus compromisos éticos y políticos, cuando logró apropiarse de una autonomía que le posibilitaba contrastar realidades y responder creativamente a los desafíos que proponían conceptualizaciones y prácticas que iban en búsqueda de una sociedad más justa.

En opinión de Olga Sanjurjo (1998), el debate teórico - práctico sobre la Pedagogía está abierto, la histórica discusión acerca del estatuto científico de la misma mantiene total vigencia en la actualidad, donde es necesario la sistematicidad y el desarrollo conceptual, entre otras razones, por lo que en diferentes medios intelectuales, se debate entre diversas paradojas tales como:

- "La necesidad social cada vez mayor de saberes acerca de la educación, mayores requerimientos/ desprestigio de su estatuto científico;

- Mayor desarrollo del campo de acción/ mayor cuestionamiento a nivel teórico desde otros campos;

- Desarrollos cada vez más críticos a la Pedagogía tradicional al interior de la disciplina/ continua la crítica a la Pedagogía identificándola exclusivamente con la corriente tradicional, impregnada de normatividad y aliada al discurso médico;

- Las Ciencias de la Educación han desplazado a la Pedagogía, pues con el avance de estas ciencias auxiliares se 
produce un proceso de autonomización de las mismas y por supuesto la dilución o desvalorización de la Pedagogía. Se la desprestigia, se la vacía de contenido y/o se la reduce al saber" (p. 59-60).

Siguiendo esta misma línea de análisis Flórez (1994) concuerda con Sanjurjo, al expresar que el objeto de la pedagogía ha sido fraccionado desde disciplinas vecinas cuyo objeto de estudio es también el ser humano, como ser cultural - la sociología, la psicología, la economía, la lingüística, la antropología, entre otras -, que se han dedicado al estudio del proceso educativo cada una desde su propia concepción teórica y metodológica, constituyendo como consecuencia una pluralidad de objetos de estudio que no solo dispersa el proceso educativo, sino incluso el ideal de un objeto pedagógico unificado, auténtico que responda a la realidad latinoamericana.

Por su parte Julia Silber (2006) afirma que la Pedagogía entró en un proceso de debilitamiento, debido a una variedad de factores tales como:

- "El control social de la práctica pedagógica en las teorías mecanicistas;

- La negación de la posibilidad de una intervención pedagógica transformadora en posturas sociológicas;

- La diseminación del campo educativo producto de su expansión y de su crecimiento científico fragmentado;

- La continuidad de una pedagogía que con un criterio más enciclopedista que reflexivo acogía desordenadamente en su ámbito todo lo que se iba produciendo en materia de educación;

- La escasa producción específicamente pedagógica;
- Y el renegar del valor de la teorización en posturas que se calificaban como progresistas desde una deformada interpretación de Paulo Freire, y que luego se anegarían en un peligroso practicismo" (p.1)

Ante lo expuesto, los autores precisan que en la actualidad se está viviendo una nueva coyuntura histórica, lo que ha permitido que el debate pedagógico recobre interés, y por ende una creciente preocupación en los ámbitos teóricos y prácticos por reivindicar esta ciencia como el saber específico sobre la educación, reclamo epistemológico que responde a las demandas que provienen de las complejas prácticas educativas actuales.

Dentro de esta misma línea de pensamiento encontramos a Edgar Ramírez (2015) pedagogo colombiano, quien en el marco del Simposio Internacional sobre Educación y Pedagogía desarrollado en Bogotá - Colombia hace un llamado de atención a todos los profesionales involucrados en el maravilloso campo de la educación y que caló hondo cuando expresó las siguientes paradojas:

- "Una facultad de educación habla de pedagogía más por sus prácticas que por sus discursos. Mejor, las prácticas de los educadores son un discurso sobre la pedagogía. Este evento, por ejemplo.

- Los profesores no se consideran pedagogos. Se cree que enseñar es transmitir un conocimiento dado y que eso basta para ser profesor (no hemos salido del transmisionismo bancario como diría Freire).

- La pedagogía dejó de ser el área de conocimiento fundante de la formación de educadores" (p.1). 
Como señalan los autores citados, la crisis epistemológica de la pedagogía no se debe tanto a la falta de investigaciones sobre esta problemática, sino a la marcada intensión de desconocer a educadores y pedagogos que desde la práctica y la teoría expuesta en sus obras han sustentado una nueva forma de hacer la educación que va en contra de los presupuestos establecidos, Paulo Freire por ejemplo, así como también al escaso debate del estatuto epistemológico de esta ciencia en las Facultades de Ciencias de la Educación.

Por lo tanto, urge la necesidad y el compromiso de educadores y pedagogos formados $y$ en proceso de formación de promover el debate epistemológico de la Pedagogía en las Facultades de Ciencias de la Educación y de nuevas investigaciones tendientes a proponer teorías y prácticas pedagógicas emancipadoras desde América Latina y para América Latina que se ajusten a las necesidades de la cultura latinoamericana (amerindia, occidental y africana), y atienda sus particularidades, singularidades, intereses, necesidades y refleje los avances ocurridos en los países que la conforman.

Entonces, si la única forma de acceder al pensamiento universal es producir un pensamiento original desde nuestras particularidades, es la educación la llamada a ser el pilar fundamental de un desarrollo sostenible de nuestros pueblos desde la mirada pedagógica de una formación propia para América Latina (Valdivieso, 2012).

\section{A MANERA DE CONCLUSIÓN}

El debate epistemológico de la Pedagogía en las Facultades de Ciencias de la Educación en las Universidades Latinoamericanas es necesario para que educadores y pedagogos conocedores de su contexto económico, político, social y cultural establezcan propuestas para que el sistema educativo en América Latina responda a las reales necesidades de la niñez y juventud y se convierta en un factor de avance y no de retroceso para la sociedad.

Para conseguir dicho propósito es importante considerar que somos el resultado de la hibridación de tres culturas y que cada una de ellas nos ha heredado características y rasgos que nos hacen particulares y únicos en este mundo. La educación y pedagogía occidental impuesta en nuestras aulas escolares ha demostrado hasta el cansancio que no responde a las necesidades e intereses de esta nueva cultura latinoamericana, que por su connotación es diferente a las demás.

Además, dentro de este debate epistemológico de la Pedagogía es imperioso considerar que somos viejos como lo afirma Botero - Uribe (2000), si computamos la antigüedad de las culturas que se han fusionado (amerindia, occidental y africana), pero al mismo tiempo somos jóvenes porque en el encuentro de tres mundos se han producido proyecciones inéditas en la historia que no han sido tomadas en cuenta, no por cuestiones geográficas, sino por cuestiones políticas y económicas, lo que ha dado como producto la colonización mental y la colonización pedagógica, robándonos a buen decir de (Wainsztok, 2011) las cuentas y los cuentos, privándonos de los relatos históricos, filosóficos, sociológicos y pedagógicos de Nuestra América.

Si a lo anterior le sumamos una formación y práctica docente también colonizada, que ha respondido al orden económico imperante, cabría entonces preguntarnos cuál es el papel que ha jugado y sigue jugando la educación y la pedagogía en la "formación" de nuestros niños y jóvenes en América Latina y qué hemos hecho los educadores y pedagogos frente a la domesticación que hemos sido objeto. 
El problema se ahonda mucho más, cuando a esta pedagogía colonizada le han quitado su espacio y naturaleza otras ciencias bajo el nombre de ciencias de la educación como la Psicología de la Educación, la Sociología de la Educación, la Antropología Educativa y demás, quienes han tratado de tener protagonismo y han fraccionado su objeto de estudio, sin entender que un enfoque interdisciplinar para el estudio del hecho educativo, es decir, un accionar a partir de la cooperación científica, permitiría el avance y desarrollo de la educación, donde la pedagogía ocupa un lugar y privilegiado, por cuanto cuestiona, interroga, indaga y problematiza de manera original el quehacer de las demás ciencias de la educación (Zambrano, 2005; Di Caudo, 2013; Ortiz, 2017 y Vera-Rojas 2019).

El error más grave a lo largo de la historia ha sido tratar de copiar y/o imitar modelos educativos y pedagógicos desde el norte o desde el viejo continente, que por su naturalidad no tienen en cuenta esas particularidades que hacen a los latinos seres únicos y excepcionales (Valdivieso, 2012). Esto ha impedido proponer y prevalecer en nuestras aulas una pedagogía propia, pensada desde nuestro contexto que permita pensar y actuar en términos autónomos, libres e independientes, por lo tanto es necesario empezar a construir a partir del debate epistemológico de esta ciencia una pedagogía emancipadora latinoamericana en función de nuestra particular historia.

\section{REFERENCIAS BIBLIOGRÁFICAS}

Botero-Uribe, D. (2000). Manifiesto del pensamiento latinoamericano. Colombia: ed. Magisterio.

Buenavilla, R. (2001). Metodología de la investigación de la vida y obra de educadores. Instituto Superior Pedagógico E. J. Varona. La Habana.

Di Caudo, M. (2013). La ciencia pedagógica: construcciones, disputas, desafíos. Revista Sophia: Colección de Filosofía de la Educación. (14), 33-50.

Flórez, R. (1994). Hacia una pedagogía del conocimiento. Bogotá, Colombia: Editorial McGRAW-HILL.

Gadotti, M. (2003). Historia de las ideas pedagógicas. Buenos Aires, Argentina: Editorial Siglo XXI editores.

Konstantinov, N. (1974). Historia de la Pedagogía. La Habana, Cuba: Editorial pueblo y Educación.

Ortiz, A. (2017). Configuración epistemológica de la pedagogía. Tendencias que han proliferado en la historia de la educación. Rev. hist.edu. latinoam., 19 (29), 165-195.

Ramírez, E. (2015). Paradojas de la Pedagogía. Simposio Internacional organizado por la Red Iberoamericana de Pedagogía. Universidad Distrital, Bogotá, Colombia. Recuperado de https://google.com/site/esquitar.

Sanjurjo, L. (1998). El estatuto científico de la pedagogía: entre la crítica y la posibilidad. Revista Innovación Educativa, (8), 59-69.

Silber, J. (2006). Reflexiones Epistemológicas sobre la Pedagogía. Recuperado de https:// es.scribd.com/doc/ 
Valdivieso, O. (2012). Ponencia: La importancia de una educación particular para América Latina. Santiago de Cali, Colombia.

Vera-Rojas, M. (2019). Tesis Doctoral. Metodología para develar la contribución de una obra pedagógica al estatuto epistemológico de la Pedagogía en el contexto latinoamericano contemporáneo. Universidad de la Habana, Cuba.

Wainsztok, C. (2011). Pedagogías Latinoamericanas. Artículo publicado en el blog del grupo Jauretche de Argentina.

Zambrano, A. (2005). Didáctica, pedagogía y saber. Bogotá, Colombia: Magisterio. 